\title{
Skeletal muscle mass estimation in Saudi adults - relationship with obesity and hypertension
}

\author{
M. M. Alkhalaf, C. A. Edwards, M. E. J. Lean and E. Combet \\ Human Nutrition, New Lister Building, School of Medicine, College of Medical, Veterinary and Life Sciences, \\ University of Glasgow, Glasgow G31 2ER, UK
}

Socioeconomic status and lifestyle (including dietary habits) have dramatically changed in Saudi Arabia (SA) over the last few decades. SA suffers from a high burden of non-communicable diseases such as obesity and hypertension. Low body muscle mass (SM) is associated with cardiovascular risk factors including arterial stiffness which may contribute to HTN ${ }^{(1)}$. The objectives of this study were to assess skeletal muscle (SM) mass in a random sample of Saudi adults, and evaluate its contribution toward hypertension.

Participants (19-60 years old) were randomly selected from the community (Riyadh city, 2013). Informed consent was collected and blood pressure, height, weight, waist and hip circumferences (WC, HC) were measured. Obesity was defined according to $\mathrm{BMI}\left(\geqslant 30 \mathrm{~kg} / \mathrm{m}^{2}\right)^{(4)}$ and WC cut-offs (gender-specific risk categories I \& II) ${ }^{(1)}$. Whole-body SM was estimated with the Al-Gindan equations $(\mathrm{E} 1, \mathrm{E} 2)^{(2)}$ : There is no population-specific cut-offs to define low SM in the Saudi populations; low SM was defined according to Chien et al ${ }^{(3)}:<8.87 \mathrm{~kg} / \mathrm{m}^{2}$ in men and $<6.42 \mathrm{~kg} / \mathrm{m}^{2}$ in women.

$$
\begin{gathered}
S M=2.89+0.255 \text { Body Weight }(\mathrm{kg})-0.175 \operatorname{Hip}(\mathrm{cm})-0.038 \operatorname{Age}(\mathrm{y})+0.118 \operatorname{Height}(\mathrm{cm}) \\
S M=39.5+0.665 \text { Body Weight }(\mathrm{kg})-0.185 \operatorname{Waist}(\mathrm{cm})-0.418 \mathrm{Hip}(\mathrm{cm})-0.08 \operatorname{Age}(\mathrm{y})
\end{gathered}
$$

The median age of participants $(\mathrm{n}=601)$ was 29 (IQR 24-38). The median SM mass was $7 \cdot 7 \mathrm{~kg} / \mathrm{m}^{2}(\mathrm{IQR} 7 \cdot 2-8 \cdot 3)$ in women and $10.7 \mathrm{~kg} / \mathrm{m}^{2}$ (IQR 9.6-12) in men. Prevalence of low SM mass was $6.5 \%$ overall, higher in males $(11.5 \%)$ than females $(2.7 \%)$,

\begin{tabular}{|c|c|c|c|c|c|c|}
\hline & \multicolumn{3}{|l|}{ Low SM * } & \multicolumn{3}{|l|}{ Normal SM * } \\
\hline & $\begin{array}{l}\text { Male }(\mathrm{n}=30) \\
\text { median }(I Q R)\end{array}$ & $\begin{array}{l}\text { Female }(\mathrm{n}=9) \\
\text { median }(I Q R)\end{array}$ & $\begin{array}{l}\text { All }(\mathrm{n}=39) \\
\text { median }(I Q R)\end{array}$ & $\begin{array}{l}\text { Male }(\mathrm{n}=231) \\
\text { median }(I Q R)\end{array}$ & $\begin{array}{l}\text { Female }(\mathrm{n}=327) \\
\text { median }(I Q R)\end{array}$ & $\begin{array}{l}\text { All }(\mathrm{n}=558) \\
\text { median }(I Q R)\end{array}$ \\
\hline Age & $31(24-40)$ & $25(23-31)$ & $29(23-36)$ & $30(25-38)$ & $28(23-39)$ & $29(24-38)$ \\
\hline Weight (kg) & $59(52-68)^{\mathrm{a}}$ & $48,43-57$ & $58(50-68)^{\mathrm{a}}$ & $83(74-93)^{\mathrm{b}}$ & $69(59-82)^{\mathrm{b}}$ & $76(63-88)^{\mathrm{b}}$ \\
\hline Height $(\mathrm{cm})$ & $172(166-174)^{\mathrm{a}}$ & $157,153-165$ & $170(161-173)^{\mathrm{a}}$ & $172(167-175)$ & $158(153-162)^{\mathrm{a}}$ & $163(157-170)^{\mathrm{b}}$ \\
\hline BMI $\left(\mathrm{kg} / \mathrm{m}^{2}\right)$ & $21(19-23)^{\mathrm{a}}$ & $18,17-23$ & $21(18-23)^{\mathrm{a}}$ & $29(25-32)^{\mathrm{b}}$ & $28(24-32)^{\mathrm{b}}$ & $28(24-32)^{\mathrm{b}}$ \\
\hline $\mathrm{WC}(\mathrm{cm})$ & $79(71-90)^{\mathrm{a}}$ & $76(67-93)$ & $78(69-89)^{\mathrm{a}}$ & $96(86-104)^{\mathrm{b}}$ & $82(72-92)^{\mathrm{a}}$ & $88(76-99)^{b}$ \\
\hline & $n / n n,(\%)^{\$}$ & $n / n n,(\%)^{8}$ & $n / n n,(\%)^{\$}$ & $n / n n,(\%)^{\$}$ & $n / n n,(\%)^{\$}$ & $n / n n,(\%)^{\$}$ \\
\hline $\mathrm{BMI} \geqslant 30$ & $1 / 95(1)$ & $1 / 123(0 \cdot 8)$ & $2 / 218(1)^{\mathrm{a}}$ & $93 / 95(98)$ & $122 / 123(99)$ & $215 / 218(98)^{b}$ \\
\hline $\mathrm{WC}>80 \mathrm{~F} / 94 \mathrm{M}$ & $3 / 130(2 \cdot 3)$ & $3 / 181(1.7)$ & $6 / 311(1.9)^{\mathrm{a}}$ & $126 / 130(97)$ & $178 / 181(98)$ & $304 / 311(98)^{b}$ \\
\hline $\mathrm{WC}>88 \mathrm{~F} / 102 \mathrm{M}$ & $2 / 69(1.5)$ & $2 / 109(1.8)$ & $4 / 178(2 \cdot 3)^{\mathrm{a}}$ & $66 / 69(96)$ & $107 / 109(98)$ & $173 / 178(97)^{\mathrm{b}}$ \\
\hline Hypertensive & $4 / 43(9 \cdot 3)$ & $1 / 68(1.5)$ & $5 / 111(4 \cdot 5)^{\mathrm{a}}$ & $39 / 43(91)$ & $67 / 68(98)$ & $106 / 111(95)^{\mathrm{b}}$ \\
\hline
\end{tabular}
$\mathrm{p}=<0.001)$. Individuals with low SM had lower body weight and lower BMI than counterparts with normal SM ( $<<0 \cdot 001)($ Table1).

* categorization according to Chien et al ${ }^{(3)}$ (low $\mathrm{SM}<8.87 \mathrm{~kg} / \mathrm{m}^{2}$ in men and $<6.42 \mathrm{~kg} / \mathrm{m}^{2}$ ); ${ }^{\$}$ : \% of the subgroup

In comparison with the normal SM group, low SM did not significantly affect the odds of having hypertension (OR $0.63,95 \%$ CI, $0 \cdot 24-1 \cdot 64, p=0 \cdot 34)$. Relatively few cases combined low SM mass and hallmark of obesity. This is somewhat surprising, however partially explained by the cut-offs used for low SM mass, derived from a Korean population ${ }^{(3)}$. This reference group may not be suitable to characterise low SM in the Saudi population, emphasising the need for further normative data to define reference levels for specific populations, including our population of interest.

1. Ferreira I et al. (2004). J Clin Endocrinol Metab 89:2632-9.

2. Al-Gindan et al. (2014). American Society for Nutrition, ajcn-070466.

3. Chien M. Y., Huang T. Y., \& Wu Y. T. (2008). Journal of the American Geriatrics Society, 56(9), 1710-1715.

4. Lean M. et al. (1995). Br Med J, 311:158-61. 